\title{
Institutional Review Board and International Field Research in Conflict Zones
}

\author{
Srobana Bhattacharya, Georgia Southern University
}

ABSTRACT Research on political conflict can benefit immensely from fieldwork. However, the Institutional Review Board (IRB) process is elaborate and daunting that discourages rather than encourages this type of research. Existing policies often are insensitive to the many uncertainties related to field research abroad, especially in conflict zones. Three reasons for this are identified in this article. First, the federal regulations to protect human subjects of social science research are most suitable for biomedical sciences. Second, there is huge gap between "procedural ethics" and "ethics in practice." Third, there is a lack of communication or dialogue between researchers and IRBs. After discussing these reasons, I offer the following suggestions: bridging the gap between the researcher and the IRB; reducing delays in the IRB approval and revision process; encouraging collaboration and dialogue among researchers; and advocating a proactive stance by academic associations.

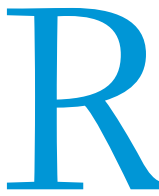
esearch on political conflict can benefit immensely from fieldwork. However, the Institutional Review Board (IRB) process can be daunting and, at times, is an impediment to such research (Carpenter 2012). IRBs have profound impact on the regulation of all research that involves "human subjects" (Marshall 2003, 270). The general purpose of an IRB-that is, protection of human subjects and promotion of ethical research-is well justified. However, the IRBs often become overzealous in their assessment of potential risks. They shut down research projects due to "perceived" rather than "actual" risks. The normative standards enforced by IRBs at US institutions are largely drawn from psychological sciences and are based on addressing problems faced by medical practitioners (Carapico 2006; Carpenter 2012). This perspective and oversight is inadequate for offering guidance to nonmedical field research involving communities and societies that are located overseas and conflict ridden. When these borrowed protocols are applied to nonmedical fields, the scrutiny process for field research in social sciences becomes lengthy and can result in a negative outcome. The process discourages scholars to continue this type of research in the future and inculcates a detrimental divide between academic research and the ethical-review process. I experienced this divide while conducting my field research in India to study the Maoist conflict.

The purpose of my field research was to determine why civilians support or do not support the left radicals, or Maoists. Thus,

Srobana Bhattacharya is an assistant professor in the department of political science at Georgia Southern University. She can be reached at sbhattacharya@georgiasouthern.edu. my initial research proposal included plans to interview civilians in both the urban and the rural areas of Eastern India. The Maoist conflict in India is not a new one but there has been a resurgence of the conflict since 2000. Since 2004, the Maoist conflict has been responsible for 10,000 deaths and has displaced another 150,000 people (Hoelscher, Miklian, and Vadlamannati 2012). The violence is sporadic and has affected primarily Central Indian regions. I chose to conduct my field research in Eastern India because of my familiarity with the region and the feasibility of actual access because the Maoist conflict resembled a low-intensity conflict in this area. In addition to civilians, I wanted to interview government officials involved in the counterinsurgency. The problem arose with "civilians"; the IRB was skeptical about the "perceived" risks associated in communicating with this segment of the population. Another problem was related to the method of attaining informed consent from civilians. Although I proposed the method of oral consent (i.e., most of the rural population in Eastern India is illiterate), the IRB would approve only written consent. When I revised the proposal to include written consent in vernacular language, the IRB was still skeptical about the risks involved and suggested that I exclude "civilians" as subjects. In my final revised proposal, I deleted civilians and included only government officials as my subjects. This most certainly changed the nature of my research. Nevertheless, it prompted me to think about future possibilities and the problems related to academic research of this nature, which escalates in the context of international field research.

Scholars in anthropology, sociology, political science, and criminology agree that there are fundamental problems in the IRB process when it is applied to international field research in the social sciences. 
After assessing their common concerns and based on my own experience related to my research project on the Maoist conflict in India, I identified three major problems in the IRB process relative to field research. First, the complex network of federal regulations that are developed to protect human subjects of social science research are most suitable for the biomedical sciences and experimental research. The regulations are flawed when applied to the social sciences because they are borrowed from the biomedical sciences (Cassell 1980). This is especially true with regard to informed consent. Second, a huge gap exists between "procedural ethics," as termed by Guillemin and Gillam (2004), and "ethics in practice." Third, there is a lack of communication or dialogue between researchers and the IRBs.

In this article, I analyze these problems and offer suggestions that may help to create a platform for understanding the IRB
Part of this rigidity was because existing regulations do not fully apply to international field research. Also, I found a general lack of knowledge among the IRB members about the local and regional cultures in Eastern India. As stated by Dolnik (2011), members of the IRB committees are rarely active researchers, and there is an almost "unofficial" norm of identifying problems with any proposal; this naturally has a perverse effect when applied to proposals on political conflict set overseas. Two of the major areas of concern are informed consent and assessment of risk. I use my specific case and experience with the IRB to illustrate common concerns and not to generalize. In many cases, IRBs reach different conclusions about similar studies (Stark 2012); therefore, it is not fair to conclude that all studies of this nature are assessed similarly.

\section{Despite my background and thorough knowledge of the regional language and culture, the IRB did not allow me to conduct interviews with the local population.}

process in general and its application to international field research in particular. Furthermore, the goal of this discussion is to reassess the ethical-review process so that it encourages rather than discourages field research overseas.

\section{PROTECTION OF HUMAN SUBJECTS IN BIOMEDICAL RESEARCH AND INTERNATIONAL FIELDWORK}

The normative standards by IRBs at US institutions are drawn primarily from the psychological sciences and are based on addressing problems faced by medical practitioners (Carapico 2006; Carpenter 2012). Cassell (1980) correctly pointed out that relationships between those who study and those who are studied are not fixed but instead vary with the type of research. The context and location of the research are important factors that affect the relationship between the researcher and those who are studied. Anthropologists and other social scientists often are concerned about how the IRBs apply an inappropriate level of scrutiny to minimal-risk research in which investigators use methods such as surveys, structured and unstructured interviews, ethnographic participant observation, oral histories, and analysis of existing datasets (Marshall 2003). At the other end of the spectrum are certain research topics-for example, political conflict, especially terrorism-that by their very nature raise questions from the human-research perspectives (Dolnik 2011). Some of the existing regulations related to obtaining informed consent are practically impossible to fulfill when conducting research on topics such as terrorism. There is a twofold problem: (1) the sensitivity of the issue, and (2) the geographical location. For my proposed fieldwork on the Maoist conflict in India, as mentioned previously, I had to make several revisions and delete certain segments of the population to be interviewed. Despite my background and thorough knowledge of the regional language and culture, the IRB did not allow me to conduct interviews with the local population. The local population was critical to my research, which analyzed levels of civilian support for insurgent groups. Even after multiple submissions and presentations of my revised documents and restating my knowledge of the area and my acquaintances there, the IRB did not allow me to continue with my original project.
In international field research, the sociocultural and political atmosphere is completely different than in the United States. The population to be studied can be illiterate or uneducated. The subjects often lack adequate knowledge to understand the entire content of the research, and they are intimidated by written documents that require a signature. This can lead to an initial mistrust between the researcher and those who would be studied. In ethnographic field research involving indirect observation, individual informed consent would be unrealistic (Marshall 2003). Many IRBs apply ethical-research principles rather mechanically following protocols that are applicable to medical research or studies that include clinical trials. A foreign country with active conflict, weak institutions, and corruption presents a different reality. These changing contexts should be considered to redefine protocols for international fieldwork. In the international context, it is important to develop culturally meaningful approaches to obtain informed consent. Obtaining consent should be a process-a series of interactions that familiarizes the subject with the researcher to understand the concept of consent (Marshall 2006). The pros and cons of using consent forms in these areas should be evaluated. When necessary, the provision of oral consent should be discussed. Kass and Hyder (2001) mentioned from personal experience that informed consent during their own research was seen as culturally insensitive but was accepted mechanically to satisfy the funding agency and government regulations. ${ }^{1}$ Hyder and Wali (2006) surveyed more than 200 researchers involved in international studies and found that $40 \%$ did not use written consent. ${ }^{2}$

The protocols as presented by the IRBs make the process of obtaining informed consent the foremost concern for ethical research. However, research ethics does not end after obtaining consent forms. There is a lack of concern about how these consents are obtained. In foreign cultures and dangerous political conditions, obtaining signatures on consent forms is difficult. Subjects, especially women, often undergo emotional stress before signing a document that is not understood. In patriarchal societies, women have little choice and their choices often are voiced through their father or husband. The review process should be sensitive to these cultural variations, assessing research protocols based on the particular sociocultural and political context. 


\section{"PROCEDURAL ETHICS" AND "ETHICS IN PRACTICE"}

Regulations to protect human subjects of social science research are a set of rules, procedures, and ethical standards that aim to protect the dignity and rights of individuals who are involved in research. It follows naturally that preexisting conditions and environments in which the individuals reside and communication procedures with which they are most familiar are recognized and protected. However, no such attempt is made by the IRBs. The ethical process becomes simply a set of rules that must be implemented. The adherence to and approval of the protocols do not safeguard ethical judgment; the protocols cannot anticipate the various dilemmas other than informed-consent and data-security issues that arise during research in conflict zones (Wood 2006). During the review process for my research, I eventually learned-as did many others in the profession-that the fundamental function of the process is not to protect human subjects but rather to protect universities from malpractice suits (Carpenter 2012).3

Guillemin and Gillam (2004) pointed out that procedural ethics alone cannot provide all that is needed for dealing with ethically important moments in qualitative research. Procedural ethics is one-dimensional, emphasizing the set of rules and their application more than an actual assessment of ethical reality. In this sense, procedural ethics becomes simply a set of technicalities. Ethics in practice, conversely, has two levels: (1) recognizing the set of ethical rules, and (2) implementing them in the context of ethical reality. Procedural ethics emphasizes the importance of individual ethics along with the set of rules. There often is a divide between "procedural ethics" and "ethics in practice." However, Guillemin and Gillam (2004) indicated a process that bridges the gap between procedural ethics and ethics in practice: that is, reflexivity. McGraw, Zvonkovic, and Walker defined reflexivity as a process whereby researchers place themselves and their practices under scrutiny, acknowledging the ethical dilemmas that permeate the research process and impinge on the creation of knowledge $(2000,68)$. Indeed, reflexivity goes a long way in understanding and evaluating the ethical purpose of the research being conducted. However, Guillemin and Gillam (2004) placed the entire onus on the researcher. The IRB also should be an active part of this reflexive process. The institution must share equal and, in some cases, greater responsibility in being reflexive about "how" individuals should be involved in research, especially in politically vulnerable areas across the world. it raises questions about ethics in the context of the researcher and the research. There is evidence of researchers who are able to conduct field research on sensitive topics internationally. This evidence indicates that IRBs vary, but it also should offer insights to successful completion of research that can serve as a model.

My research analyzed civilian support for the Maoist rebel groups in India. To collect data, I needed interviews and oral histories from civilians residing in the peripheries of Maoist-affected areas. I had definite advantages in conducting this research because I am Indian and I resided in the same location for several years. Also, I had acquaintances in the government and police departments, who conveyed their interest in providing security for me; they also helped me in the initial selection of areas (i.e., predominantly low-conflict zones). Despite having these resources and regularly updating the IRB and sharing information about them, I could not conduct my initial research project. However, it was difficult to convey this to the IRB about my research project, and it continually failed to acknowledge my expertise in the area of study and my knowledge of the geographical location. After several revisions, I could include only government officials as my interviewees. I spent several painstaking hours carefully selecting specific government officials for my interviews. In addition to government documents and newspaper archives, I expanded my secondary-document analysis to include rare journal entries from ex-activists and Maoist prisoners. I also communicated with several scholars who had prior field-research experience abroad in conflict zones and similar locations; most of them quickly understood the issues and warned me about the IRB process. A few shared their approved research protocols with me to demonstrate that research in conflict zones, riskier than the location I chose, was indeed possible. 4 Subsequently, I shared these protocols with the IRB, but this did not immediately help facilitate the approval process of my research.

The communication gap between the researcher and the IRB should not be a reason to impede social science research. The IRBs should expand their ethical concerns to include the issue of a communication gap between the researcher and the committee. The communication gap is heightened by the fact that few committee members have actual knowledge about several geographical areas chosen for fieldwork. Also, they do not have adequate information about particular topics and they apply general rules to evaluate a research proposal.

\section{Unlike experimental research, field-research design is concerned with access to settings, people, and research-relevant documents.}

\section{COMMUNICATION GAP BETWEEN THE RESEARCHER AND THE IRB}

One of the main problems in the ethical-review process is the lack of communication and dialogue between the researcher and the IRB. To increase my understanding of the process, I communicated with IRB members even before I started writing the proposal. I asked questions and sought guidelines. However, other than the reiteration of standard protocols, I learned little from these interactions. After the initial rejection of my proposal, I met in person with the committee chair to discuss the prospect of my research and revisions to my proposal. During the conversation, the feasibility of the research was questioned and, on several occasions, I had to repeat the justification for my topic. Although my experience is specific to only one institution,

\section{ASSESSING INTERNATIONAL FIELD RESEARCH}

Unlike experimental research, field-research design is concerned with access to settings, people, and research-relevant documents. For this reason, it often requires amending the research design to accommodate sudden changes and/or unavailability of certain people (Yanow and Schwartz-Shea 2008). Not many ethnographers start with specific hypotheses that later will be systematically tested. As a primary data-gathering technique, ethnographers often rely on the relationships that they have established with potential subjects (Bosk and de Vries 2004).

International field research is fraught with uncertainties that are specific to a region and the sociopolitical context within the region. This leads to significant differences between experimental research 
and field research. The differences have implications for three of the fundamental IRB processes: informed consent, understanding potential harms and risks, and confidentiality. For instance, observation of public behavior as a part of ethnographic research does not necessarily require informed consent (Marshall 2003). 5 Furthermore, for various reasons, ethnographers cannot particularly inform the subjects of the risks and benefits of cooperating. In developing countries, the mere mention of benefits-especially in rural areas-is related to monetary incentives. After a researcher convinces the subjects that there are no financial benefits for them or their families, there is general disappointment. ${ }^{6}$ This behavioral shift can have a direct impact on further communication with the researcher. Also, discussing the risks from an academic perspective is too difficult for the subjects to understand. Before delving into directed questions related to the research, the process of explaining risk and benefits may deter the subjects from establishing a rapport with the researcher. In addition, it is difficult to specify risks because the findings are not known (Bosk and de Vries, 2004).

In many non-Western countries, the concept of ethical review for the social sciences is relatively unexplored and few mechanisms are in place to oversee or approve any proposed field research. At times, IRBs in the United States propose that a researcher obtain a "go-ahead" from the country where the research will be conducted. In nonmedical fields, this approval from the foreign country is almost impossible. The administrative setup varies among countries, which often is ignored by IRBs. These uncertainties are real problems that must be discussed at length. Instead, IRBs are more concerned with "the procedural and bureaucratic" (Bosk and de Vries 2004, 260).

Indeed, there are serious ethical dilemmas when conducting research in international regions that have either a history of conflict or an ongoing conflict. Does this automatically lead to the conclusion that all ethnographic research in conflict areas is to be abandoned? "Fieldwork is possible even in the most dangerous contexts" (Sluka 1995, 293). Fortunately, several researchers have successfully completed field research in conflict zones; however, almost all of them raise concerns about the IRB process. Again, the fundamental issue in conducting research in conflict zones is related to uncertainties and heightened by the possible occurrence of violence. This issue can be resolved in the following ways: careful selection of the location (i.e., not all areas are equally violence prone), careful selection of subjects, choice of questions to be asked, and method of observation. Another aspect is the breadth of knowledge of the researchers and their academic, is important to learn about causes and consequences of complex issues (e.g., political conflict). These recommendations are not restricted to ethnographic field research in conflict zones but rather field research in general. They include (1) bridging the gap between the researcher and IRB members, (2) reducing delays in the IRB approval and revision process, (3) encouraging collaboration and dialogue among researchers, and (4) advocating a proactive stance by academic associations.

\section{Bridging the Gap between the Researcher and IRB Members}

Many scholars have voiced the demand for a more informed IRB (Bosk and deVries 2004; Marshall 2003). These studies focused on the following issues: encouraging and improving studies on how IRB works; increasing the participation of social scientists and anthropologists in IRBs; and educating IRB members. In addition, I recommend a dialogue and a series of interactions between IRB members and researchers. These interactions should occur in three phases: before submission of the protocol, during the research process, and after the research is completed. This will help the IRB to understand the full scope of the research, with specific focus on the actual experiences of the researcher in the field and the outcome of the research. This will be beneficial in an IRB's assessment of similar future research projects. Furthermore, these processes will raise an IRB's awareness of the many "actual" challenges and successes encountered during field studies.

\section{Reducing Delays in the IRB Approval and Revision Process} Delays occur when IRB approval stalls the onset of a project (Bosk and de Vries 2004). The lengthy process of original submission, revisions, further objections, and subsequent resubmission is a frustrating experience for many field researchers. In addition, research topics that are considered potentially risky (e.g., political conflict, violence against women, and criminal behavior) in many cases require a separate process. This type of research is categorized as Type III, which requires lengthy committee meetings. In many institutions, these meetings are scheduled either once a month or fortnightly. Invariably, the research protocols need revisions, but each resubmission becomes a monthlong case or, if a researcher is fortunate, perhaps two weeks. Also, multiple revisions are required to obtain final approval. In comparison, the approval process for research topics that are not potentially risky typically is fairly rapid. For sensitive topics, institutions should plan to implement faster appeals mechanisms. Again, for these issues,

\section{Few scholars have mentioned the possibility of collaboration between and among qualitative researchers with regard to ameliorating the IRB process and field research in general. This type of collaboration has tremendous potential.}

professional, or family ties to the region to be studied-all of which helped in my research on Maoist conflict in Eastern India.

\section{RECOMMENDATIONS}

The tensions in the IRB process cannot be resolved by a single set of actions (Fujii 2012). I recommend a multilayered course of action in which both the researcher and the IRB members engage actively to encourage ethnographic field research in general and international field research involving challenging topics in particular. This research institutions should assign members who are either familiar with the type of research or have experience in the topics.

\section{Encouraging Collaboration and Dialogue among Researchers}

Few scholars have mentioned the possibility of collaboration between and among qualitative researchers with regard to ameliorating the IRB process and field research in general. This type of collaboration has tremendous potential. Field researchers should share 
their experiences with the IRB process to help identify common problems. Furthermore, IRBs can issue guidelines for designing research protocols. This collaboration is especially important for international field research in conflict zones. Researchers with substantial experience in this type of field research can share information about the IRB process. In addition, they can discuss their actual research experience with those who are planning international travel to conduct similar research in the same or a nearby location. This will provide an encouraging academic environment for sharing problems as well as successes, which is pertinent for creating a constructive research environment. There are several listservs for qualitative researchers, and this network can include one based on international field research.

\section{Advocating a Proactive Stance by Academic Associations}

Yanow and Schwartz-Shea (2008) discuss specific political associations (e.g., APSA) that are actively involved in IRB policy reforms. Furthermore, they specifically state APSA's need to collaborate with other organizations within the Consortium of Social Science Associations to work together and provide informational and educational services for both IRB members and researchers. This is instrumental for improving the existing process. Furthermore, other academic organizations related to the humanities and the liberal arts should be involved in the process as well. To encourage field research in political conflict, the International Studies Association, Women in Conflict Studies, and Peace Science Society should conduct workshops and symposia during annual meetings. Furthermore, these associations provide an excellent platform for researchers to share their experiences and discuss future projects. The purpose of these collaborations across associations should be both policy- and academic-oriented, with the aim of improving IRB policies and developing appropriate regulatory policies that encourage rather than discourage field research.

\section{NOTES}

1. Kass and Hyder (2001) had this experience in Latin America with those of limited reading ability. This segment of the population was hesitant about signing documents. I had a similar experience during my field research at a tea plantation in rural Eastern India.

2. In the survey, Hyder and Wali (2006) found that scientific and medical researchers were more likely to use written consent, whereas nonmedical researchers used written consent only when the subjects were highly literate.

3. During the review process of my field research, I communicated with several social scientists. All of them reiterated the fact that the fundamental aim of an IRB is to protect a university from malpractice suits. They further mentioned that it is almost impossible to obtain IRB approval for projects that involve research in conflict zones. These scholars also shared concerns about lack of training and knowledge among IRB members to assess conditions for international field research.

4. I particularly want to mention the role of Women in Conflict Studies in helping me locate researchers who have conducted field research abroad. I eventually communicated with Dara K. Cohen, who shared the protocol for her research conducted in Sierra Leone.

5. In fact, Marshall (2003) considers the feasibility of consent in this setting to be unrealistic. She agrees, however, that there is a need for informed consent in certain situations involving direct observation of group activities (e.g., nurses in a hospital setting).

6. Fujji (2012) mentions that subjects often acknowledge that they understand there will be no payment but still may count on benefits yet to come. In Rwanda, she interviewed prisoners in 2004. Many of the prisoners, knowing that there will be no financial benefits, continue to ask for her help in legal matters.

\section{R E F E R E N C E S}

Bosk, Charles L., and Raymond G. de Vries. 2004. "Bureaucracies of Mass Deception Institutional Review Boards and the Ethics of Ethnographic Research.” The Annals of the American Academy of Political and Social Science 595 (1): 249-63.

Carapico, Sheila. 2006. "No Easy Answers: The Ethics of Field Research in the Arab World.” PS: Political Science and Politics 39 (3): 429-31.

Carpenter, Charli. 2012. "You Talk of Terrible Things So Matter-of-Factly in This Language of Science: Constructing Human Rights in the Academy." Perspectives on Politics 10 (2): 363-83.

Cassell, Joan. 1980. "Ethical Principles for Conducting Fieldwork." American Anthropologist 82 (1): 28-41.

Dolnik, Adam. 2011. "Conducting Field Research on Terrorism: A Brief Primer." Perspectives on Terrorism 5 (2):3-36.

Fujii, Lee Ann. 2012. "Research Ethics 101: Dilemmas and Responsibilities." PS: Political Science and Politics 45 (4): 717-23.

Guillemin, Marilys, and Lynn Gillam. 2004. "Ethics, Reflexivity, and 'Ethically Important Moments' in Research." Qualitative Inquiry 10 (2): 261-80.

Hoelscher, Kristian, Jason Miklian, and K. C. Vadlamannati. 2012. "Hearts and Mines: A District Level Analysis of the Maoist Conflict in India." International Studies Review 15 (2): 141-6o.

Hyder, Adnan A., and Salman A. Wali. 2006. "Informed Consent and Collaborative Research: Perspectives from the Developing world." Developing World Bioethics 6 (1): 33-40.

Kass, Nancy E., and Adnan Ali Hyder. 2001. "Attitudes and Experiences of U.S and Developing Country Investigators Regarding U.S. Human Subjects Regulations." In Ethical and Policy Issues in International Research:Clinical Trials in Developing Countries, Vols. I and II. Bethesda, MD: National Bioethics Advisory Commission, B-1-220.

Marshall, Patricia A. 2003. "Human Subjects Protections, Institutional Review Boards, and Cultural Anthropological Research." Anthropological Quarterly $76(2): 269-85$.

. 2006. "Informed Consent in International Health Research." Journal of Empirical Research on Human Research Ethics 1 (1): 25-42.

McGraw, Lori A., Anisa M. Zvonkovic, and Alexis J. Walker. 20oo. "Studying Postmodern Families: A Feminist Analysis of Ethical Tensions in Work and Family Research." Journal of Marriage and Family 62 (1): 68-77.

Sluka, Jeffrey A. 1995. "Reflection on Managing Danger in Fieldwork: Dangerous Anthropology in Belfast." In Fieldwork under Fire: Contemporary Studies of Violence and Survival, ed. Carolyn Nordstorm and Antonius C. G. M. Robben, 276-94. Berkeley: University of California Press.

Stark, Laura. 2012. Behind Closed Doors: IRBs and the Making of Ethical Research. Chicago: University of Chicago Press.

Wood, Elisabeth Jean. 20o6. "The Ethical Challenges of Field Research in Conflict Zones." Qualitative Sociology 29 (3): 373-86.

Yanow, Dvora, and Peregrine Schwartz-Shea. 2008. "Reforming Institutional Review Board Policy: Issues in Implementation and Field Research." PS: Political Science and Politics 41 (3): 483-94. 